\title{
An Ultrasensitive Flexible and Bending Sensor for Detecting
} Angle and Micro-force

\author{
Yang Chen ${ }^{1, \mathrm{a}}$, Shaofei Guo ${ }^{1, \mathrm{~b}}$,Yan Xiong ${ }^{1, \mathrm{c}}$ and Lina Hao ${ }^{1, \mathrm{~d}^{*}}$ \\ ${ }^{1}$ School of Mechanical Engineering and Automation, Northeastern University, Shenyang, China \\ aemail: neuchenyang@163.com, bemail: 1303718029@qq.com, email: 477065@qq.com, ${ }^{\text {'}}$ email: \\ haolina@me.neu.edu.cn
}

Keywords: Flexible and bending sensor, Angle displacement sensor, Micro-force sensor Abstract. The dexterous robotic hand is required to investigate the characteristic of finger movements with perception ability of various sensors. A good angle displacement sensor can well describe finger motion in robotic hand manipulation tasks. In this paper, a flexible and bending sensor which was suitable to be mounted on flexible joint of robotic hand was presented in application of detecting angle displacement. Design of condition circuit was conducted to analyze the relationship between output voltage and bending angle. Calibration of the sensor results exhibited significant liner relationship, and the sensitivity of the sensor was $31.68 \mathrm{~V} /{ }^{\circ}$ functioned as angle displacement sensor. In addition, a Load Cells Force/Torque micro-force sensor was employed to calibrate the sensor which could also be used as a micro-force sensor in application of cell micro-manipulation filed, and the sensitivity of the sensor was $31.31 \mathrm{mV} / \mathrm{mN}$ functioned as micro-force sensor.

\section{Introduction}

In order to make the robot more intelligent and manipulating tasks well like human beings, most researches had been done on the perception system of robot and interaction with human beings and unstructured environment [1]-[3]. A dexterous prosthesis hand is one of the most vital components in robot, and the perception ability of the end manipulator is a key specification for the robot. A well designed robotic hand always has multi-degree of freedom (DOF) like human hand, so we should get the degrees of each finger joints in real time to well understand the behavior of manipulating tasks, and according to the obtained data from joint sensors, the feedback signal is generated by control procedure to form a closed loop. Nowadays a few researchers have paid close attention to adaptive manipulation hand with flexible joint, so some perception sensors on joint degree and torque have been discussed deeply. The overview of literatures on this study is as follows.

F. Lotti et al [4] proposed the piezoresistive bend sensors in measuring the 2-DOF articulation of the upper fingers. Because of existence of loss of sensor sensitivity, it could not measure the lateral bending and compression. A. Cavallo et al [5] measured the position of flexible joints with optoelectronic sensors, so did Leif P. Jentoft et al [6]. The difference between [5] and [6] is that the former hand was single-DOF and the latter one is multi-DOF. However the disadvantages of optoelectronic sensors are signal alteration and attenuation due to bending or misalignment. Leif $\mathrm{P}$. Jentoft and Robert D. Howe [7] proposed a low-cost flexible joint measuring by a Hall-effect sensor for their low drift and the ease with assembling on the SDM process, while the sensors will work incorrectly by unexpected magnetic field, so the robotic hand cannot work in a magnetic field environment. Nazrul Hamizi Adnan et al [8]-[9] employed a variable resistance output bend sensor in Data Glove system to acquire the motion information of finger joint. The experiment results well verified the effectiveness of sensors. M. Hazwan Ali et al [10] used carbon resistivity element inside thin strip flex sensor whose resistance varied from variation of the joint degree.

In this paper, we used the ultrasensitive hybrid carbon polymer-based piezoresistive (HCP) film which was the same as the sensor in reference [11] as a flexible joint sensor. The HCP films performed as a sensitive and durable 3D micro-force sensor in Luo's work [11]. However, we innovatively proposed that this sensitive flexible sensor could be mounted on a flexible joint to measure its position, and the flexibility of the sensor could make its shape compliance to joint pose consistently, and it was in favor of data acquisition and processing in real time. 
This paper is organized as follows: The second section gives a brief property introduction of HCP film, design of a half-bridge condition circuit to get output voltage of flexible sensor. The third section presents the calibration of flexible sensor which can be used as both micro-force sensor and angle displacement sensor, and regression analysis of data significant demonstrates effectiveness of the sensor. The last section is the summary on the work of this paper.

\section{Sensitive Flexible Sensor}

\section{HCP Film}

The piezoresistive sensing film presents a continuous variable resistance intensified with bending process. It is ultrasensitive reaction to the perception area with length of $14 \mathrm{~mm}$, width of $2 \mathrm{~mm}$, and thickness of $0.14 \mathrm{~mm}$ because of the carbon polymer material inside entire thin strip with length of 20 $\mathrm{mm}$, width of $6 \mathrm{~mm}$, and thickness of $0.14 \mathrm{~mm}$. This flexible sensor is also cost-effective, stainless, durable and light weight, so it becomes an optimal position sensor for flexible joint in application of robotic hand. In addition, the thermal drift of the flexible sensor is as low as $1.63 \%$ in the temperature range between $22^{\circ} \mathrm{C}$ and $75.1^{\circ} \mathrm{C}$ [11]. That is to say, the flexible sensor can be applied without room temperature environment. The flexible sensor employed in our study is shown in Fig. 1.

\section{Design of Condition Circuit}

The flexible sensor works as a two-variable-series resistance with bending motion process. The two variable resistances are measurable from pin 1 to pin 2 and from pin 3 to pin 2 respectively. The two variable resistances should be same in initial state in theory. However, the ultrasensitive ability of the flexible sensor leads to difference of the two resistances, and unnecessary bending also make the resistance values different. Based on the basic information of the flexible sensor, we have designed a half-bridge condition circuit shown in Fig. 2 to measure voltage outputs of the flexible sensor. The nulling resistor is used in half-bridge condition circuit to balance the different resistances in the flexible sensor, so that the output voltage is zero. The gain resistance $R_{G}$ of this circuit is a constant value $42.54 \mathrm{~K} \Omega$, and then the output voltage gain can be calculated by equation (1).

$$
G=\frac{49.4 k \Omega}{R_{G}}+1
$$

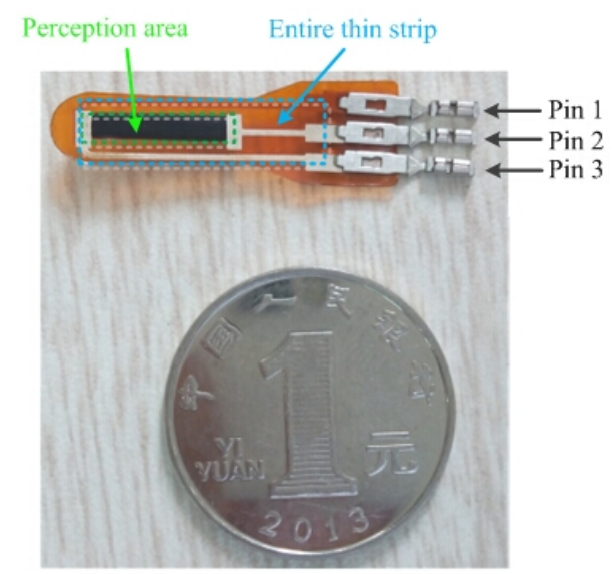

Fig. 1 The flexible sensor

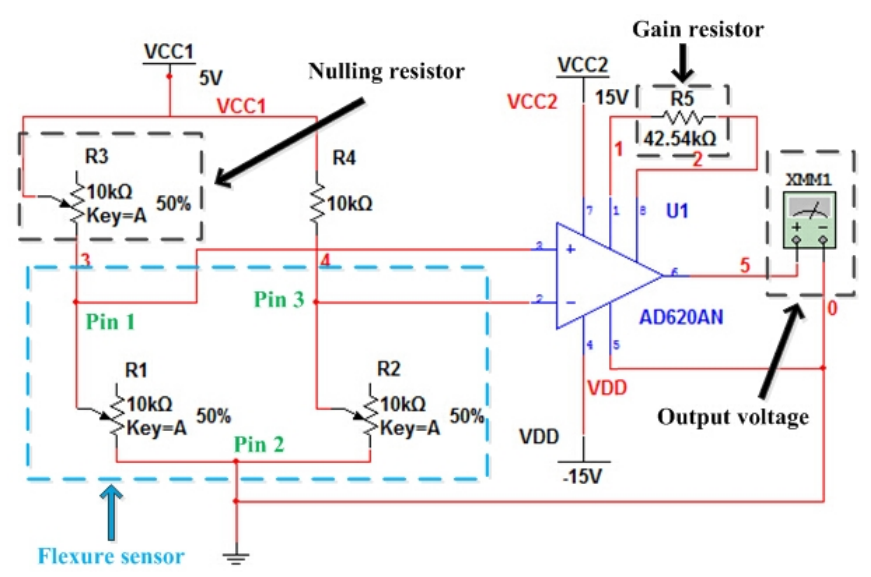

Fig. 2 The half-bridge condition circuit

\section{Experiments}

\section{Calibration of Flexible Sensor}

Before the flexible sensor can be applied in a robotic hand, deflection-voltage and micro-force-voltage calibration were conducted by a Load Cells Force/Torque micro-force sensor (Measuring range: $0 \sim 10 \mathrm{~g}$, resolution: $15 \mu \mathrm{N}$ ), NI-PCI6221 DAQ (16 bits ADC, output voltage range: $\pm 10 \mathrm{~V}$ ) and 5-DOF micro-positioning platform (X, Y and $\mathrm{Z}$ orientations: $10 \mu \mathrm{m} / \mathrm{step}), \mathrm{PC}$, DC power 
and some other common experimental apparatus. The calibration experimental platform is represented in Fig. 3.

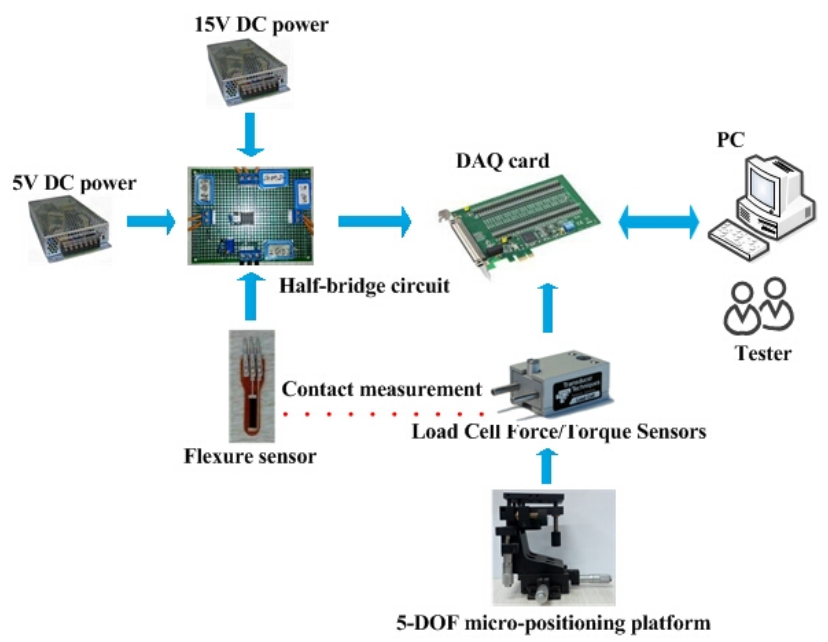

Fig. 3 The calibration experimental platform

In the calibration, the Load Cells Force/Torque micro-force sensor which was calibrated before with the measure $9.24 \mathrm{mN} / \mathrm{V}$ is attached to 5-DOF micro-positioning platform, and the flexible sensor is attached to magnetic stand. The tip of flexible sensor perception area is perpendicular to the micro-force sensor contact. The nulling resistor should be adjusted to make output voltage zero. The calibration begins at above initial position, and making the micro-force sensor move 20 steps towards the flexible sensor bending orientation each time, and recording the output voltages of micro-force sensor and flexible sensor.

\section{Regression Analysis of Data}

Regression Analysis can be used to predict relationship between micro-force and voltage of flexible sensor functioned as a micro-force sensor and between step distance and voltage of flexible sensor functioned as an angle displacement sensor. We utilize the experiment data above, and it is easy to find that linear polynomial is suitable to express relationship between tip displacement and voltage of flexible sensor shown in Fig. 4 and relationship between tip displacement and force of micro-force sensor shown in Fig. 5. The curve-fitted equations (2) and (3) of Fig. 4 and 5 are as follows.

$$
\begin{aligned}
& U=0.0726 d-0.0014 \\
& F_{1}=2.3185 d+0.0445
\end{aligned}
$$

Simultaneous equations (2) and (3) can obtain relationship between force and voltage of flexible sensor, whose expression is shown as equation (4).

$$
F_{2}=31.9353 U+0.0892
$$

where $d(\mathrm{~mm})$ represents step distance, $U(\mathrm{~V})$ represents output voltage of flexible sensor, $F_{1}(\mathrm{mN})$ represents force of micro-force sensor and $F_{2}(\mathrm{mN})$ represents force of flexible sensor. So we get sensitivity of this flexible sensor functioned as a micro-force sensor (sensitivity: $31.31 \mathrm{mV} / \mathrm{mN}$ ). However, we want to use this flexible sensor as an angle displacement sensor. To make the problem simplification, in small deformation of flexible sensor situation which is shown in Fig. 6, the bending angle of flexible sensor can be calculated by equation (5).

$$
\theta=\frac{180 d}{\pi R}
$$

where $R$ (approximate $25 \mathrm{~mm}$ ) represents approximate to length of flexible sensor and $\theta\left(^{\circ}\right.$ ) represents bending angle of flexible sensor. Simultaneous equations (2) and (5) can obtain relationship between angle and voltage of flexible sensor, whose expression is shown as equation (6). 
$\theta=31.57 U+0.04$

We can also get the sensitivity $31.68 \mathrm{~V} /{ }^{\circ}$ of this flexible sensor functioned as an angle displacement sensor. In order to verify the flexible sensor is good enough to function as both micro-force sensor and angle displacement sensor, correlation coefficients of equations (2) to (3) need to be calculated. Correlation coefficient can indicate linearity relationship between independent variable and dependent variable. We get the values of correlation coefficient from equation (7). Where $X_{\mathrm{i}}$ represents independent variable, $Y_{\mathrm{i}}$ represents dependent variable, $\bar{X}$ represents mean of independent variable, $\bar{Y}$ represents mean of dependent variable and $r^{2}$ represents correlation coefficient. $r$ are 0.9980 and 0.9945 which give a significant proof to liner relationship.

$$
r^{2}=\frac{\sum_{i=1}^{n}\left(X_{i}-\bar{X}\right)\left(Y_{i}-\bar{Y}\right)}{\sum_{i=1}^{n}\left(X_{i}-\bar{X}\right)^{2} \sum_{i=1}^{n}\left(Y_{i}-\bar{Y}\right)^{2}}
$$

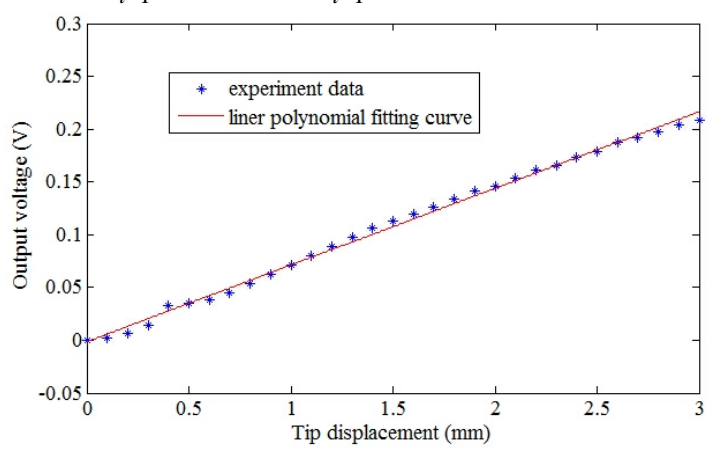

Fig. 4 Relationship between tip displacement and Fig. 5 Relationship between tip displacement and voltage of flexible sensor

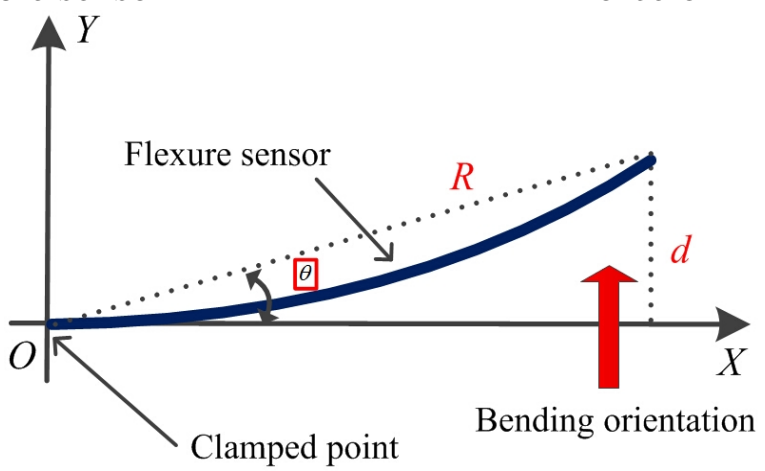

Fig. 6 Schematic diagram of flexible sensor bending

\section{Summary}

An ultrasensitive flexible and bending sensor with the condition circuit has been analysis in detecting angle and micro-force, which can well describe a compliant joint angle as an angle displacement sensor and detect micro-force in cell injection manipulation. The sensitivity of the sensor functioned as detecting angle displacement is $31.68 \mathrm{~V} /{ }^{\circ}$, and the sensitivity of the sensor functioned as detecting angle displacement was $31.31 \mathrm{mV} / \mathrm{mN}$. Because of the ultrasensitive static characteristics, this piezoresistive sensing film can be employed in many other fields.

\section{Acknowledgements}

This work was particularly sponsored by the National High Technology Research and Development Program of China (863 Program) under Grant No. 2015AA042302, the Equipment Pre-Research Program of China under Grant No. 62501040412, the National Natural Science Foundation of China under Grant No. 61573093 and the University Innovation Team of Liaoning Province (LT2014006). 
The authors also thank sincerely the reviewers and editors for their very pertinent remarks that helped this article become clearer and more precise.

\section{References}

[1] M. Beetz, F. Balint-Benczedi, N. Blodow, D. Nyga, T. Wiedemeyer and Z. C. Marton. RoboSherlock: Unstructured Information Processing for Robot Perception. In: IEEE International Conference on Robotics and Automation (ICRA), Seattle, Washington, USA. 2015.

[2] K. Ramirez-Amaro, M. Beetz, G. Cheng. Understanding the intention of human activities through semantic perception: observation, understanding and execution on a humanoid robot. Advanced Robotics, 2015, 29(5): 345-362.

[3] S. B. Schorr, F. Q. Zhan, W. R. Provancher, A. M. Okamura. Environment Perception in the Presence of Kinesthetic or Tactile Guidance Virtual Fixtures, In: Proceedings of the Tenth Annual ACM/IEEE International Conference on Human-Robot Interaction. ACM New,York, NY, USA, 2015, 287-294.

[4] F. Lotti, P. Tiezzi, G. Vassura, L. Biagiotti, G. Palli and C. Melchiorri. Development of UB hand 3: Early results, In: Proceedings of the 2005 IEEE International Conference on Robotics and Automation, Barcelona, 2005, 4488-4493.

[5] A. Cavallo, G. De Maria, C. Natale, S. Pirozzi. Optoelectronic joint angle sensor for robotic fingers. Sensors and Actuators A: Physical, 2009, 152(2): 203-210.

[6] L. P. Jentoft, A. M. Dollar, C. R. Wagner, R. D. Howe. Intrinsic Embedded Sensors for Polymeric Mechatronics: Flexible and Force Sensing. Sensors, 2014, 14(3): 3861-3870.

[7] L. P. Jentoft, R. D. Howe. Compliant fingers make simple sensors smart, Proceedings of the 2010 IFToMM/ASME Workshop on Underactuated Grasping (UG2010), Montreal, Canada. 2010, 19.

[8] N. H. Adnan, K. Wan, A. B. Shahriman, S. K. Zaaba and H. Desa. The Development of a Low Cost Data Glove by Using Flexible Bend Sensor for Resistive Interfaces, In: The 2nd International Malaysia-Ireland Joint Symposium on Engineering, Science and Business. 2012, 579-587.

[9] N. H. Adnan, K. Wan, A. B. Shahriman, et al. Measurement of the flexible bending force of the index and middle fingers for virtual interaction, Procedia Engineering, 2012, 41, 388-394.

[10] M. H. Ali, K. Wan, Y. C. Seah, N. H. Adnan, J. A. Bakar. Analysis of Finger Movement for Robotic Hand (MAPRoh-1) by Using Motion Capture and Flexible Bend Sensor, The 8th International Conference on Robotic, Vision, Signal Processing \& Power Applications. Springer Singapore, 2014, 23-29.

[11] Y. Luo, Y. Shen, N. Mohan, Durable and cost-effective 3-D microforce sensor for musical tuning enhanced micro palpation of biological entities, SENSORS, 2013 IEEE. IEEE, 2013, 1-4. 